\title{
An Optimal Streamline Diffusion Finite Element Method for a Singularly Perturbed Problem
}

\author{
Long Chen and Jinchao Xu
}

\begin{abstract}
The stability and accuracy of a streamline diffusion finite element method (SDFEM) on arbitrary grids applied to a linear 1-d singularly perturbed problem are studied in this paper. With a special choice of the stabilization quadratic bubble function, the SDFEM is shown to have an optimal second order in the sense that $\left\|u-u_{h}\right\|_{\infty} \leq C \inf _{v_{h} \in V^{h}}\left\|u-v_{h}\right\|_{\infty}$, where $u_{h}$ is the SDFEM approximation of the exact solution $u$ and $V_{h}$ is the linear finite element space. With the quasi-optimal interpolation error estimate, quasi-optimal convergence results for the SDFEM are obtained. As a consequence, an open question about the optimal choice of the monitor function for a second order scheme in the moving mesh method is answered.
\end{abstract}

\section{Introduction}

In this paper, we shall analyze the optimality of a specially designed streamline diffusion finite element method for the singularly perturbed problem

$$
\begin{aligned}
& -\varepsilon u^{\prime \prime}-b(x) u^{\prime}=f \text { in }(0,1), \\
& u(0)=u(1)=0,
\end{aligned}
$$

where the positive diffusion constant $\varepsilon$ satisfies $0<\varepsilon \ll b_{0} \leq b(x), \forall x \in[0,1]$ and $b$ is sufficiently smooth. We do not assume $f$ is smooth which means the equation (1.1) should be understood in the distributional sense for general $f$.

It is well known that the standard finite element method (FEM) on quasiuniform grids is not uniformly stable with respect to $\varepsilon$. The error bounds presented in the classic error analysis of FEM become meaningless when $\varepsilon$ goes to zero. Therefore we are looking for so-called $\varepsilon$-uniform methods where the stability and accuracy are independent of $\varepsilon$. The most popular method for the convection-dominated problem is the streamline diffusion finite element method (SDFEM) introduced by Hughes et. al. [19]. The SDFEM is quite satisfactory for practical situations and many convergence estimates have been done in the literature [5, 20,21, 26, 28, 31,33, 36, 37]. It is, however, very hard to prove the SDFEM is $\varepsilon$-uniformly optimal in the $L^{\infty}$

2000 Mathematics Subject Classification. 65G99, 65N30, 76R99.

This work was supported in part by NSF DMS-0209497 and NSF DMS-0215392 and the Changjiang Professorship through Peking University. 
norm on arbitrary grids even for the one dimensional linear singularly perturbed problem $[31,32]$.

In our recent work [11], when the convection coefficient $b$ is constant, we proved that a specially designed SDFEM approximation $u_{h}$ is quasi-optimal in the sense that

$$
\left\|u-u_{h}\right\|_{\infty} \leq C \inf _{v_{h} \in V^{h}}\left\|u-v_{h}\right\|_{\infty}
$$

where $u$ is the solution of singularly perturbed problem (1.1)-(1.2), $u_{h}$ is the numeric solution obtained by the SDFEM and $V^{h}$ is the piecewise linear and global continuous finite element space. We would like to explicitly point out that here $C$ is a constant that is independent of both $\varepsilon$ and $N$ where $N$ is the number of grid elements. In this paper, we extend the estimate (1.3) to a slightly more general variable coefficients $b(x)$. Furthermore, we shall establish this estimate by means of discrete Green functions which is different from the approach used in [11].

Thanks to (1.3) the convergence of the SDFEM becomes an approximation problem which is well studied in the literature (see, e.g. $[9,15])$. If the grid is quasiuniform under the metric $\left|u^{\prime \prime}\right|^{1 / 2}$, we are able to get a quasi-optimal interpolation error estimate, namely $\left\|u-u_{I}\right\| \leq C\left\|u^{\prime \prime}\right\|_{1 / 2} N^{-2}$, where $\left\|u^{\prime \prime}\right\|_{1 / 2}:=\left(\int_{0}^{1}\left|u^{\prime \prime}\right|^{1 / 2} d x\right)^{2}$. One commonly used approach to construct such a quasiuniform grid under some metric is the use of monitor function and equidistribution principal $[4,12,13,29]$.

Many people use the arc-length as the monitor function, namely $M=\sqrt{1+\left|u^{\prime}\right|^{2}}$ or its discrete analogue $[12,13,24,29]$. The first order uniform convergence on the equidistributed grid is obtained even for the fully adapted algorithm [13,24]. In this sense, an optimal choice of monitor function for the first order scheme is the arc-length.

By our theory if the grid is obtained by equidistributing $\left|u^{\prime \prime}\right|^{1 / 2}$, the SDFEM approximation to the solution of (1.1)-(1.2), with a careful choice of the stabilization function, has a quasi-optimal second order convergence, namely

$$
\left\|u-u_{h}\right\|_{\infty} \leq C\left\|u^{\prime \prime}\right\|_{1 / 2} N^{-2} .
$$

(1.4) is the most desirable estimate we may expect to obtain for problem (1.1)-(1.2). Thus an optimal choice of the monitor function is $\left|u^{\prime \prime}\right|^{1 / 2}$. Note that $\left\|u^{\prime \prime}\right\|_{1 / 2}$ is $\varepsilon$ uniformly bounded in many cases, the convergence (1.4) is indeed $\varepsilon$-uniform. The uniform convergence of two special a priori grids, Bakhvalov and Shishkin grids can be easily obtained in this framework [11].

The layout of the rest of this paper is as follows: in the next section we will describe the standard FEM and introduce the SDFEM. In Section 3, we will make use of discrete Green functions to prove the $\left(l_{\infty}, w_{-1, \infty}\right)$ stability of the SDFEM. In Section 4 , we will show the truncation error in $w_{-1, \infty}$ norm is bounded by $\left\|u-u_{I}\right\|_{\infty}$. As a consequence we obtain the quasi-optimality of SDFEM. In Section 5, we will recall a quasi-optimal interpolation error estimate to prove the convergences of SDFEM on some a priori grids.

\section{The streamline diffusion finite element method}

In this section, we shall introduce the SDFEM for the singularly perturbed problem (1.1)-(1.2) and our special choice of the stabilization function. 
Let $H^{1}=\left\{v \in L^{2}, v^{\prime} \in L^{2}\right\}$ and $H_{0}^{1}=\left\{v \in H^{1}, v(0)=v(1)=0\right\}$. The weak solution to (1.1)-(1.2) is a function $u \in H_{0}^{1}$ such that

$$
a(u, v)=(f, v), \forall v \in H_{0}^{1},
$$

where $(\cdot, \cdot)$ is the $L^{2}$ inner product and $a(u, v)=\varepsilon\left(u^{\prime}, v^{\prime}\right)-\left(b u^{\prime}, v\right)$. The existence and uniqueness of the weak solution are easy to establish.

For a positive integer $N$, let $\mathcal{T}_{N}=\left\{x_{i} \mid 0=x_{0}<x_{1}<\ldots<x_{N+1}=1\right\}$ be an arbitrary grid, $\tau_{i}$ denote the element $\left[x_{i-1}, x_{i}\right]$ and $h_{i}=x_{i}-x_{i-1}$. We denote by $\varphi_{i}(x)$ the nodal basis function at point $x_{i}$ and the finite element space $V^{h}=\left\{v_{h} \mid v_{h}=\sum_{i=0}^{N} v_{i} \varphi_{i}(x)\right\}$. The standard finite element discretization of (2.1) is to find a $u_{h} \in V^{h}$ such that $u_{h}(0)=u_{h}(1)=0$ and

$$
a\left(u_{h}, v_{h}\right)=\left(f, v_{h}\right), \forall v_{h} \in V^{h} \cap H_{0}^{1} .
$$

To introduce the SDFEM, we first modify our weak form to be

$$
\tilde{a}(u, v):=a(u, v)-\sum_{i=1}^{N} \int_{x_{i-1}}^{x_{i}} \delta_{i}\left(-\varepsilon u^{\prime \prime}-b u^{\prime}\right) b v^{\prime},
$$

where $\delta_{i}$ is a stabilization function in $\left[x_{i-1}, x_{i}\right]$. We will discuss the choice of $\delta_{i}$ in a moment. For the exact solution $u$ of (1.1)-(1.2), it satisfies

$$
\tilde{a}(u, v)=\tilde{f}(v), \text { for all } v \in H_{0}^{1},
$$

where $\tilde{f}(v)=(f, v)-\sum_{k=1}^{N} \int_{x_{k-1}}^{x_{k}} \delta_{k} f b v^{\prime}$. The SDFEM is to find $u_{h} \in V^{h}$ such that

$$
\tilde{a}\left(u_{h}, v_{h}\right)=\tilde{f}\left(v_{h}\right), \forall v_{h} \in V^{h} .
$$

With a slightly abuse of the notation, we still use $u_{h}$ to denote the vector $\left(u_{h}\left(x_{1}\right), \ldots, u_{h}\left(x_{N}\right)\right) \in \mathbb{R}^{N}$. Taking $v_{h}=\varphi_{i}$ in $(2.2)$, we obtain an algebraic equation $A u_{h}=f_{h}$, where $A=\left(a_{i j}\right)$ is a tri-diagonal matrix and $\left(f_{h}\right)_{i}=\tilde{f}\left(\varphi_{i}\right)$. Direct calculation gives us that

$$
\begin{aligned}
a_{i, i-1} & =-\frac{\varepsilon}{h_{i}}+\frac{\int_{x_{i-1}}^{x_{i}} b \varphi_{i}}{h_{i}}-\frac{\int_{x_{i-1}}^{x_{i}} \delta_{i} b^{2}}{h_{i}^{2}}, \\
a_{i, i} & =\frac{\varepsilon}{h_{i}}+\frac{\varepsilon}{h_{i+1}}-\frac{\int_{x_{i-1}}^{x_{i}} b \varphi_{i}}{h_{i}}+\frac{\int_{x_{i}}^{x_{i+1}} b \varphi_{i}}{h_{i+1}}+\frac{\int_{x_{i-1}}^{x_{i}} \delta_{i} b^{2}}{h_{i}^{2}}+\frac{\int_{x_{i}}^{x_{i+1}} \delta_{i+1} b^{2}}{h_{i+1}^{2}}, \\
a_{i, i+1} & =-\frac{\varepsilon}{h_{i+1}}-\frac{\int_{x_{i}}^{x_{i+1}} b \varphi_{i}}{h_{i+1}}-\frac{\int_{x_{i}}^{x_{i+1}} \delta_{i+1} b^{2}}{h_{i+1}^{2}},
\end{aligned}
$$

with standard modifications for $i=1$ and $i=N$.

The choice of the stabilization function $\delta_{i}$ is the key point in our paper. We choose

$$
\delta_{i}=\kappa_{i} h_{i} \varphi_{i} \varphi_{i-1}
$$

where

$$
\kappa_{i}=\left\{\begin{array}{cccc}
\varepsilon_{i}^{-1} h_{i} & \text { if } & \varepsilon>\int_{x_{i-1}}^{x_{i}} b \varphi_{i}, \\
\left(\int_{x_{i-1}}^{x_{i}} b^{2} \varphi_{i} \varphi_{i-1}\right)^{-1} \int_{x_{i-1}}^{x_{i}} b \varphi_{i} & \text { if } & \varepsilon \leq \int_{x_{i-1}}^{x_{i}} b \varphi_{i} .
\end{array}\right.
$$

Lemma 2.1. If $\delta_{i}$ is determined by (2.3), $A$ is an M-matrix. 
ProOF. With the choice $(2.3)$, it is easy to see $a_{i, i-1}<0$. Since $a_{i, i+1}<0$ and $a_{i, i-1}+a_{i, i}+a_{i, i+1}=0$, we know $a_{i, i}>0$ and $a_{1,1}>a_{1,2}$. Thus $A$ is an M-matrix.

In recent years, it is shown that the SDFEM can be derived from more general fundamental principles such as residual-free bubble finite element method [6,17]. The choice of the stabilization function can be chosen as an approximation of a special local bubble function on each element. Our choice of $\delta_{i}$ is a quadratic bubble function.

\section{Stability of the SDFEM}

In this section we shall prove the $\left(l_{\infty}, w_{-1, \infty}\right)$ stability $[1,25]$ of the SDFEM which is a discrete analogue of the $\left(L_{\infty}, W_{-1, \infty}\right)$ stability of the continuous differential operator [13,24]. For the terminology, we refer to [25] and [23]. Here we follow $[1,25]$ to use the discrete Green functions.

The $i$-th discrete Green function $G^{i}$ is a function in $V_{h}$ satisfying $G^{i}(0)=$ $G^{i}(1)=0$ and

$$
\tilde{a}\left(\varphi_{j}, G^{i}\right)=\delta_{j}^{i}
$$

where $\delta_{i}^{j}$ is the Kronecker symbol.

Lemma 3.1. Let $G^{i}=\sum_{j=1}^{N} G_{j}^{i} \varphi_{j}$, we have the following properties of $G_{j}^{i}$.

(1) $G_{j}^{i} \geq 0,1 \leq i, j \leq N$

(2) $0 \leq G_{1}^{i}<\ldots<G_{i}^{i}>G_{i+1}^{i}>\ldots>G_{N}^{i}$.

(3) $G_{i}^{i} \leq b_{0}^{-1}$.

ProOF. (1) and (2) easily follow from the property that $A$ is an M-matrix. For (3), we first note that (3.1) implies that for any $v_{h} \in V_{h}, \tilde{a}\left(v_{h}, G^{i}\right)=v_{h}\left(x_{i}\right)$. Taking $v_{h}=\sum_{j=1}^{i} \varphi_{j}$, we get

$$
\sum_{j=1}^{N} \sum_{k=1}^{i} a_{j, k} G_{j}^{i}=1 .
$$

Since $a_{j, j-1}+a_{j, j}+a_{j, j+1}=0$, we can simplify (3.2) to

$$
\left(a_{1,1}+a_{1,2}\right) G_{1}^{i}+a_{i, i+1}\left(G_{i+1}^{i}-G_{i}^{i}\right)+\left(a_{i-1, i}+a_{i, i}+a_{i, i+1}\right) G_{i}^{i}=1 .
$$

Note that, by (1) and (2), the first two terms in the left side are positive and

$$
a_{i-1, i}+a_{i, i}+a_{i, i+1}=\frac{1}{h_{i+1}} \int_{x_{i}}^{x_{i+1}} b \geq b_{0} .
$$

Therefore $G_{i}^{i} \leq b_{0}^{-1}$.

LEMMA 3.2. The SDFEM with $\delta_{i}$ determined by (2.3) applied to any grid is $\left(l_{\infty}, w_{-1, \infty}\right)$ uniform stable in the sense that

$$
\left\|v_{h}\right\|_{\infty} \leq \frac{2}{b_{0}} \max _{j=1, \ldots, N}\left|\sum_{k=j}^{N}\left(A v_{h}\right)_{k}\right|, \forall v_{h} \in V_{h} .
$$


ProOF

$$
\begin{aligned}
v_{h}\left(x_{i}\right) & =\tilde{a}\left(v_{h}, G^{i}\right)=\sum_{j=1}^{N} G_{j}^{i} \tilde{a}\left(v_{h}, \varphi_{j}\right) \\
& =\sum_{j=1}^{N} G_{j}^{i}\left(A v_{h}\right)_{j}=\sum_{j=1}^{N}\left[\left(G_{j-1}^{i}-G_{j}^{i}\right) \sum_{k=j}^{N}\left(A v_{h}\right)_{k}\right] .
\end{aligned}
$$

Therefore we have

$$
\begin{aligned}
\left\|v_{h}\right\|_{\infty} & \leq \sum_{j=1}^{N}\left|G_{j-1}^{i}-G_{j}^{i}\right| \max _{j=1, . .,}\left|\sum_{k=j}^{N}\left(A v_{h}\right)_{k}\right| \\
& \leq \max _{i=1, \ldots, N} 2 G_{i}^{i} \max _{j=1, \ldots, N}\left|\sum_{k=j}^{N}\left(A v_{h}\right)_{k}\right| \\
& \leq \frac{2}{b_{0}} \max _{j=1, . ., N}\left|\sum_{k=j}^{N}\left(A v_{h}\right)_{k}\right|
\end{aligned}
$$

\section{Quasi-optimality of the SDFEM}

In this section, we shall analyze the truncation error of the SDFEM in the $l_{-1, \infty}$ norm and thus get the quasi-optimality of the SDFEM.

For a given grid $\mathcal{T}_{N}$, let $u_{I}$ be the linear interpolant of $u$ and $e(x)=\left(u_{I}-\right.$ $\left.u_{h}\right)(x)=\sum e_{i} \varphi_{i}$ with $e_{i}=e\left(x_{i}\right), i=1,2, \ldots, N$. Since $\tilde{a}\left(u-u_{h}, v_{h}\right)=0$, we obtain the error equation

$$
\begin{aligned}
& \tilde{a}\left(e, \varphi_{i}\right)=\tilde{a}\left(u_{I}-u, \varphi_{i}\right), i=1,2, \ldots, N, \\
& e_{0}=e_{N+1}=0 .
\end{aligned}
$$

The right side $\left(\tilde{a}\left(u_{I}-u, \varphi_{i}\right)\right)$ corresponds to the truncation error in the finite difference method. A direct calculation gives us

LEMMA 4.1.

$$
\tilde{a}\left(u_{I}-u, \varphi_{i}\right)=t_{i}-t_{i+1}+s_{i}
$$

where

$$
\begin{aligned}
t_{i} & =\frac{1}{h_{i}} \int_{x_{i-1}}^{x_{i}}-b\left(u_{I}-u\right) \\
& +\frac{\varepsilon}{h_{i}} \int_{x_{i-1}}^{x_{i}} b \delta_{i}\left(u_{I}-u\right)^{\prime \prime} \\
& +\frac{1}{h_{i}} \int_{x_{i-1}}^{x_{i}} b^{2} \delta_{i}\left(u_{I}-u\right)^{\prime}
\end{aligned}
$$

and

$$
s_{i}=-\int_{x_{i-1}}^{x_{i}} b^{\prime}\left(u_{I}-u\right) \varphi_{i} .
$$


Lemma 4.2. Assume $b \in W^{2,1}$, then

$$
\sum_{i=1}^{N}\left|s_{i}\right| \leq C\left\|u-u_{I}\right\|_{\infty}, \text { and }\left\|t_{i}\right\|_{\infty} \leq C\left\|u-u_{I}\right\|_{\infty}
$$

ProOF. First

$$
\sum_{i=1}^{N}\left|s_{i}\right| \leq\left\|u-u_{I}\right\|_{\infty} \sum_{i=1}^{N} \int_{x_{i-1}}^{x_{i}}\left|b^{\prime}\right|=|b|_{1,1}\left\|u-u_{I}\right\|_{\infty} .
$$

For (4.3),

$$
\left|\frac{1}{h_{i}} \int_{x_{i-1}}^{x_{i}} b\left(u_{I}-u\right)\right| \leq\left\|u-u_{I}\right\|_{\infty}\|b\|_{\infty}
$$

For (4.4)

$$
\frac{\varepsilon}{h_{i}} \int_{x_{i-1}}^{x_{i}} b \delta_{i}\left(u_{I}-u\right)^{\prime \prime}=\frac{\kappa_{i} \varepsilon}{h_{i}} \frac{1}{h_{i}} \int_{x_{i-1}}^{x_{i}} b\left(x-x_{i-1}\right)\left(x_{i}-x\right)\left(u_{I}-u\right)^{\prime \prime} .
$$

If $\varepsilon \geq \int_{x_{i-1}}^{x_{i}} b \varphi_{i-1}$, then $\kappa_{i} \varepsilon / h_{i}=1$. Otherwise,

Thus we only need to bound

$$
\frac{\kappa_{i} \varepsilon}{h_{i}} \leq\|b\|_{\infty} \kappa_{i} \leq\left(\frac{\|b\|_{\infty}}{b_{0}}\right)^{2} .
$$

$$
\begin{aligned}
& \left|\frac{1}{h_{i}} \int_{x_{i-1}}^{x_{i}} b\left(x-x_{i-1}\right)\left(x_{i}-x\right)\left(u_{I}-u\right)^{\prime \prime}\right| \\
= & \left|\frac{1}{h_{i}} \int_{x_{i-1}}^{x_{i}}\left[b\left(x-x_{i-1}\right)\left(x_{i}-x\right)\right]^{\prime \prime}\left(u_{I}-u\right)\right| \\
\leq & \left\|u-u_{I}\right\|_{\infty} \frac{1}{h_{i}} \int_{x_{i-1}}^{x_{i}}\left|\left[b\left(x-x_{i-1}\right)\left(x_{i}-x\right)\right]^{\prime \prime}\right| .
\end{aligned}
$$

Now we estimate

$$
\begin{aligned}
I & =\frac{1}{h_{i}} \int_{x_{i-1}}^{x_{i}}\left|\left[b\left(x-x_{i-1}\right)\left(x_{i}-x\right)\right]^{\prime \prime}\right| \\
& \leq \frac{1}{h_{i}} \int_{x_{i-1}}^{x_{i}}\left(\left|b^{\prime \prime}\left(x-x_{i-1}\right)\left(x_{i}-x\right)\right|+\left|b^{\prime}\left[\left(x-x_{i-1}\right)\left(x_{i}-x\right)\right]^{\prime}\right|+|2 b|\right) \\
& =I_{1}+I_{2}+I_{3} .
\end{aligned}
$$

It is easy to get

$$
\begin{array}{r}
\left|I_{1}\right| \leq h_{i} \int_{x_{i-1}}^{x_{i}}\left|b^{\prime \prime}\right| \leq\|b\|_{2,1}, \\
\left|I_{2}\right| \leq \int_{x_{i-1}}^{x_{i}}\left|b^{\prime}\right| \leq|b|_{1,1} \text {, and }\left|I_{3}\right| \leq 2\|b\|_{\infty} .
\end{array}
$$

For (4.5), following the same pattern, we obtain

$$
\left|\frac{1}{h_{i}} \int_{x_{i-1}}^{x_{i}} b^{2} \delta_{i}\left(u_{I}-u\right)^{\prime}\right| \leq\|b\|_{\infty}\left(\|b\|_{\infty}+|b|_{1,1}\right)\left\|u-u_{I}\right\|_{\infty} .
$$

Combining these estimates together and the fact that $W^{2,1}$ is embedded into $L^{\infty}$, we finish the proof. 
With Theorem 3.2 and Lemma 4.2 we can bound the discretization error by the interpolation error.

LEMMA 4.3.

$$
\left\|u-u_{h}\right\|_{\infty} \leq C\left\|u-u_{I}\right\|_{\infty}
$$

Proof. By Lemma 3.2, we have

$$
\begin{aligned}
\left\|u_{I}-u_{h}\right\|_{\infty} & \leq C \max _{j=1, \ldots, N}\left|\sum_{k=j}^{N}\left(A\left(u_{I}-u_{h}\right)\right)_{k}\right| \\
& =C \max _{j=1, \ldots, N}\left|\sum_{k=j}^{N} \tilde{a}\left(u_{I}-u, \varphi_{k}\right)\right| \\
& \leq C\left(\max _{j=1, . ., N}\left|t_{j}-t_{N}\right|+\left|\sum_{i=1}^{N} s_{i}\right|\right) \\
& \leq C\left(2\left\|t_{i}\right\|_{\infty}+\sum_{i=1}^{N}\left|s_{i}\right|\right) \\
& \leq C\left\|u-u_{I}\right\|_{\infty} .
\end{aligned}
$$

The desired result follows from the triangle inequality.

THEOREM 4.4. Let $u_{h}$ be the SDFEM approximation to the solution of equations (1.1)-(1.2) with stabilization function $\delta_{i}$ determined by (2.3), we have

$$
\left\|u-u_{h}\right\|_{\infty} \leq C \inf _{v_{h} \in V^{h}}\left\|u-v_{h}\right\|_{\infty}
$$

Proof. Let us define an operator $P_{h}: H_{0}^{1} \rightarrow V_{h}$ by $P_{h} u=u_{h}$. By Lemma 4.2,

$$
\left\|u-u_{h}\right\|_{\infty} \leq C\left\|u-u_{I}\right\|_{\infty} \leq C\left(\|u\|_{\infty}+\left\|u_{I}\right\|_{\infty}\right) \leq C\|u\|_{\infty} .
$$

Thus

$$
\left\|P_{h} u\right\|_{\infty}=\left\|u_{h}\right\|_{\infty} \leq\|u\|_{\infty}+\left\|u-u_{h}\right\|_{\infty} \leq C\|u\|_{\infty}
$$

With the property $P_{h}^{2}=P_{h}$, for any $v_{h} \in V^{h}$, we have

$$
\left\|u-u_{h}\right\|_{\infty}=\left\|\left(I-P_{h}\right)\left(u-v_{h}\right)\right\|_{\infty} \leq C\left\|u-v_{h}\right\|_{\infty} .
$$

Since it is true for any $v_{h} \in V_{0}^{h}$, we finish the proof.

\section{Convergence of the SDFEM}

In this section, we shall first recall an interpolation error estimate from $[14,15]$ and then present a general convergence result for the SDFEM. We shall make use of the a priori bound of the $\left|u^{\prime \prime}\right|$ for the problem (1.1)-(1.2) to construct a priori quasi-optimal grid which yields a $\varepsilon$-uniform second order convergence.

Given a function $u \in C^{2}[0,1]$, a function $H(x)$ is called a majorizing second derivative of $u$, if $\left|u^{\prime \prime}(x)\right| \leq H(x), x \in(0,1)$. With this majorizing second derivative, we can define a new metric on $[0,1]$ by $H$. For an element $\tau_{i} \in \mathcal{T}$, its length in the metric $H$ are denoted by $\left|\tau_{i}\right|_{H}$, namely $\left|\tau_{i}\right|_{H}=\int_{x_{i-1}}^{x_{i}} H^{1 / 2}(x) d x$. The basic assumptions we need are

(A1) $H$ is monotone in each element $\tau_{i}, \quad i=1,2, \ldots, N+1$. 
(A2) $\left|\tau_{i}\right|_{H}$ is almost equidistributed in the sense that

$$
\max _{i}\left|\tau_{i}\right|_{H} \leq \frac{\beta}{N} \sum_{i=1}^{N+1}\left|\tau_{i}\right|_{H} .
$$

THEOREM 5.1. Let $u \in C^{2}[0,1]$ and the mesh $\mathcal{T}_{N}$ satisfy assumptions (A1) and (A2), the following error estimate holds:

$$
\left\|u-u_{I}\right\|_{\infty} \leq C\|H\|_{1 / 2} N^{-2} .
$$

REMARK 5.2. This error estimate is optimal in the sense that for a strictly convex (or concave) function, the above inequality holds asymptotically in a reversed direction for $H=\left|u^{\prime \prime}\right|[9]$.

In our recent work [9], we have developed a general interpolation error estimate in any spatial dimension and for general $L^{p}$ norm. In high dimensions, the new metric is given by a scaling, which depends on $p$ and dimensions $n$, of the majorizing Hessian matrix of the approximated function $u$. The monotonicity in the assumption (A1) is replaced by no oscillation assumption. For details, we refer to our recent work [7-10].

THEOREM 5.3. Let $u_{h}$ be the SDFEM approximation to the solution to (1.1)(1.2) on a grid satisfying assumptions (A1) and (A2), and $\delta_{i}$ is determined by (2.3), then

$$
\left\|u-u_{h}\right\|_{\infty} \leq C\|H\|_{1 / 2} N^{-2} .
$$

Proof. It is an immediate consequence of Lemma 4.3 and Theorem 5.1.

To construct an appropriate layer-adapted grids, the key is to get a majorizing second derivative. Fortunately for most singularly perturbed problems in one dimension we do have a priori bound of $\left|u^{\prime \prime}\right|$ which happens to be monotone. The following a priori bound of the derivatives of the solution is well known in the literature. See for example [22,30] or [27].

LEMMA 5.4. Let $u$ be the solution to equation (1.1)-(1.2) with smooth source term $f$, we have a priori bound for $u^{(k)}, k=0,1,2$ that is

$$
\left|u^{(k)}(x)\right| \leq 1+\varepsilon^{-k} e^{-b_{0} x / \varepsilon}, k=0,1,2 \quad \forall x \in[0,1] .
$$

Based on this majorizing second derivative, a quasi-optimal mesh is obtained by using the following monitor function

$$
M=H^{1 / 2}=\left(1+\varepsilon^{-2} e^{-b_{0} x / \varepsilon}\right)^{1 / 2} .
$$

COROLlary 5.5. The SDFEM approximation with $\delta_{i}$ determined by (2.3) to (1.1)-(1.2) with smooth right side $f$ on the grid obtained by the equidistribution of the monitor function $M$ in (5.2) will give a uniform second order approximation $u_{h}$, namely

$$
\left\|u-u_{h}\right\|_{\infty} \leq C N^{-2} \text {. }
$$

Many existing layer-adapted grids, including Shishkin grids and Bakhvalov grids, can be thought as approximations of the quasi-optimal grids by using simple approximations of the monitor function (5.2). Convergences of our SDFEM on those grids can be found at [11].

We would like to point out that Theorem 5.3 can be also applied to non-smooth data $f$. If we can get a priori information of the second derivative and adapt the 
grid to capture the singularity of the solution, then we can get the second order uniform convergence. For example, the following equation is considered in [31] (with a different sign of the convection coefficient).

$$
\begin{aligned}
& -\varepsilon u^{\prime \prime}-b(x) u^{\prime}=f+\delta(\cdot-d) \text { on } \Omega^{-} \cup \Omega^{+}, \\
& u(0)=u(1)=0,
\end{aligned}
$$

where $\Omega=(0,1), d \in \Omega, \Omega^{-}=(0, d), \Omega^{+}=(d, 1)$ and $\delta(\cdot-d)$ denotes the Diracdelta function at point $d$. Function $f$ is sufficiently smooth on $\bar{\Omega}$. The equation (5.3)-(5.4) should be understood in the distribution sense and it is well known that it has a unique solution $u \in H_{0}^{1}(\Omega)$ which has an exponential interior layer at $x=d$ and boundary layer at $x=0$. Furthermore, the following a priori estimate of the second derivative can be found at [31].

$$
\begin{aligned}
& \left|u^{(k)}(x)\right| \leq C\left(1+\varepsilon^{-k} e^{-b_{0} x / \varepsilon}\right), x \in \Omega^{-}, k=0,1,2,3 . \\
& \left|u^{(k)}(x)\right| \leq C\left(1+\varepsilon^{-k} e^{-b(x-d) / \varepsilon}\right), x \in \Omega^{+}, k=0,1,2,3 .
\end{aligned}
$$

With this information we can construct the corresponding layer-adapted grid to obtain the optimal convergence rate of the SDFEM. This example illustrate the usefulness of the quasi-optimality of the SDFEM considering the fact that results for singularly perturbed problem with discontinuous right-hand side are relatively rare $[16,31]$.

\section{Conclusion and further remarks}

In this paper, we have shown the quasi-optimality of a specially designed SDFEM for a singularly perturbed problem. With a priori information of the second derivative of the solution, we could construct a mesh to obtain an optimal second order convergence.

In practice, $u^{\prime \prime}$ is unknown. Basically we have two ways to prove the convergence of a fully adapted algorithm. One is to derive a posteriori error estimate for our optimal SDFEM using the framework in [23]. It is interesting to note that the estimate (4.4) in [23] for a different second-order method is a discrete version of the estimate given by Theorem 5.3. Another approach is to recovery $u^{\prime \prime}$ from the numerical solution, in which the superconvergence theory will play an important role. On superconvergent recovery schemes, we refer to Zikienwicz-Zhu [38, 39], Hoffmann-Schatz-Wahlbin-Wittum [18], Zhang-Naga [35], Bank-Xu [2,3], and XuZhang [34] etc. The difficulty is again the $\varepsilon$-uniformity. If $\varepsilon$ is too small while the initial grid is coarse. It is hopeless to get a reasonable information of $u^{\prime \prime}$ from the recovery scheme. Therefore it is desirable to have a robust way to construct a mesh which satisfies conditions (A1) and (A2). In [8], we developed a multilevel homotopic adaptive methods for convection dominated problems in two dimensions. This paper can be seen as the first step of the mathematical justification of our algorithm.

\section{References}

1. V. B. Andreev, The green function and a priori estimates of solutions of monotone threepoint singularly perturbed finite-difference schemes, Differential Equation 37 (2001), no. 7, 923-933.

2. R. E. Bank and J. Xu, Asymptotically exact a posteriori error estimators, Part I: Grids with superconvergence, SIAM J. Numer. Anal. 41 (2003), no. 6, 2294-2312. 
3. _ Asymptotically exact a posteriori error estimators, Part II: General unstructured grids, SIAM J. Numer. Anal. 41 (2003), no. 6, 2313-2332.

4. G. Beckett and J. A. Mackenzie, Convergence analysis of finite difference approximations on equidistributed grids to a singularly perturbed boundary value problem, Applied Numerical Mathematics 35 (2000), 87-109.

5. F. Brezzi, T. J. R. Hughes, L. D. Marini, A. Russo, and E. Süli, A priori error analysis of residual-free bubbles for advection-diffusion problems, SIAM J. Numer. Anal. 36 (1999), no. 4, 1933-1948.

6. F. Brezzi and A. Russo, Choosing bubbles for advection-diffusion problems, Mathematical Models and Methods in Applied Science 4 (1994), 571-587.

7. L. Chen, Mesh smoothing schemes based on optimal Delaunay triangulations, 13th International Meshing Roundtable, Sandia National Laboratories, 2004, pp. 109-120.

8. L. Chen, P. Sun, and J. Xu, Multilevel homotopic adaptive finite element methods for convection dominated problems, The Proceedings for 15th Conferences for Domain Decomposition Methods, Lecture Notes in Computational Science and Engineering, vol. 40, Springer, 2004, pp. 459-468.

9. __ Optimal anisotropic simplicial meshes for minimizing interpolation errors in $L^{p}$ norm, Accepted by Math. Comp., 2004.

10. L. Chen and J. Xu, Optimal Delaunay triangulations, Journal of Computational Mathematics 22 (2004), no. 2, 299-308.

11. _ Stability and accuracy of adapted finite element methods for singularly perturbed problems, Submitted to Numer. Math., 2004.

12. Y. Chen, Uniform pointwise convergence for a singularly perturbed problem using arc-length equidistribution, J. Comput. Appl. Math. 159 (2003), no. 1, 25-34.

13. Uniform convergence analysis of finite difference approximations for singular perturbation problems on an adaptive grid, Accepted by Advances in Computational Mathematics, 2004.

14. C. de Boor, Good approximation by splines with variable knots, Int. Seines Numer. Math, Birkhauser Verlag, Basel 21 (1973), 57-72.

15. R. A. Devore and G. G. Lorentz, Constructive approximation, NY:Spring-Verlag, New York, 1993.

16. P. A. Farrell, A. F. Hegarty, J. J. H. Miller, E. O'Riordan, and G. I. Shishkin, Singularly perturbed convection-diffusion problems with boundary and weak interior layers, Journal of Computational and Applied Mathematics 166 (2004), 131-151.

17. L. P. Franca and A. Russo, Deriving upwinding, mass lumping and selective reduced integration by residual-free bubbles, Applied Mathematics Letters 9 (1996), 83-88.

18. W. Hoffmann, A. H. Schatz, L. B. Wahlbin, and G. Wittum, Asymptotically exact a posteriori estimators for the pointwise gradient error on each element in irregular meshes I: A smooth problem and globally quasi-uniform meshes, Math. Comp. 70 (2001), 897-909.

19. T. J. R. Hughes and A. Brooks, A multidimensional upwind scheme with no crosswind diffusion, Finite Element Methods for Convection Dominated Flows (T. J. R. Hughes, ed.), ASME, vol. 34, New York, 1979, pp. 19-35.

20. C. Johnson and U. Nävert, An analysis of some finite element methods for advection-diffusion problems, Analytical and Numerical Approaches to Asymptotic Problems in Analysis (A. v. d. S. O. Axelsson and L. S. Frank, eds.), Amsterdam, NorthHolland, 1981, pp. 99-116.

21. C. Johnson, A. H. Schatz, and L. B. Wahlbin, Crosswind smear and pointwise errors in streamline diffusion finite element methods, Math. Comp. 49 (1987), 25-38.

22. R. B. Kellogg and A. Tsan, Analysis of some difference approximations for a singular perturbation problem without turning points, Math. Comput 32 (1978), 1025-1039.

23. N. V. Kopteva, Maximum norm a posteriori error estimates for a one-dimensional convectiondiffusion problem, SIAM J. Numer. Anal. 39 (2001), no. 2, 423-441.

24. N. V. Kopteva and M. Stynes, A robust adaptive method for quasi-linear one-dimensional convection-diffusion problem, SIAM J. Numer. Anal. 39 (2001), 1446-1467.

25. T. Linß, Layer-adapted meshes for convectioni-diffusion problems, Comput. Methods Appl. Mech. Engrg. 192 (2003), 1061-1105.

26. T. Linßand M. Stynes, The SDFEM on Shishkin meshes for linear convection-diffusion problems, Numer. Math. 87 (2001), 457-484. 
27. J. J. H. Miller, E. O'Riordan, and G. I. Shishkin, Fitted numerical methods for singular perturbation problems, World Scientific, 1996.

28. K. Niijima, Pointwise error estimates for a streamline diffusion finite element scheme, Numer. Math 56 (1990), 707-719.

29. Y. Qiu, D. M. Sloan, and T. Tang, Convergence analysis of an adaptive finite difference method for a singular perturbation problem, J. Comput. Appl. Math. 116 (2000), 121-143.

30. H. G. Roos, M. Stynes, and L. Tobiska, Numerical methods for singularly perturbed differential equations, Springer series in Computational Mathematics, vol. 24, Springer Verlag, 1996.

31. H. G. Roos and H. Zarin, The streamline-diffusion method for a convection-diffusion problem with a point source, J. Comput. Appl. Math. 150 (2003), 109-128.

32. G. Sangalli, Quasi optimality of the supg method for the one-dimensional adavection-diffusion problem, SIAM J. Numer. Anal. 41 (2003), no. 4, 1528-1542.

33. M. Stynes and L. Tobiska, The SDFEM for a convection-diffusion problem with a boundary layer: Optimal error analysis and enhancement of accuracy, SIAM J. Numer. Anal. 41 (2003), no. $5,1620-1642$.

34. J. Xu and Z. M. Zhang, Analysis of recovery type a posteriori error estimators for mildly structured grids, Math. Comp. (2003), 781-801.

35. Z. M. Zhang and A. Naga, A new finite element gradient recovery method: Superconvergence property, accepted by SIAM J. Sci. Comput.

36. G. Zhou, How accurate is the streamline diffusion finite element method?, Math. Comp. 66 (1997), no. 217, 31-44.

37. G. Zhou and R. Rannacher, Pointwise superconvergence of the streamline diffusion finite element method, Numer. Meth. PDEs 12 (1996), 123-145.

38. O. C. Zienkiewicz and J. Z. Zhu, The superconvergence patch recovery and a posteriori error estimates. Part 1: The recovery techniques, Internat. J. Numer. Methods Engrg. 33 (1992), $1331-1364$.

39. - The superconvergence patch recovery and a posteriori error estimates. Part 2: Error estimates and adaptivity, Internat. J. Numer. Methods Engrg. 33 (1992), 1365-1382.

Department of Mathematics, Pennsylvania State University, USA

E-mail address: chenlong@psu.edu

The School of Mathematical Science, Peking University, China, and Department of Mathematics, Pennsylvania State University, USA

E-mail address: xu@math.psu.edu 